\title{
A Prediction Method for Gas Turbine Overhaul Makespan with Uncertainties
}

\author{
Tao Li, Zhen Chen, and Yusong Liu
}

\begin{abstract}
The prediction of a gas turbine overhaul makespan benefits much from the real-time supervision of overhaul process and fast response to abnormal conditions, which ensure the product delivery in time for customers. Previous research indicated that many uncertainties contribute to the inaccurate of overhaul process and thus lead to delivery delay. A novel prediction method compromised with Program Evaluation and Review Technique (PERT) and Monte Carlo simulation is proposed to solve this issue. The uncertain factors affecting the overhaul makespan are investigated first, then the generic overhaul process modelling is produced to understand the relationship and constraints between detailed processes. Based on that, the duration time distribution of the overhaul makespan plans can be generated. It is possible to finalize the prediction method considering the distribution of overhaul time probability and critical work path, and the process dependences. A gas turbine is used as a case study to examine this method. The results show it is advanced in the efficiency compared with traditional methods, and has the potential to meet the requirements of high demand overhaul service for airliners.
\end{abstract}

Keywords Gas turbine overhaul - Uncertainty - PERT · Makespan prediction · Simulation 